\title{
Prenatal Infection Affects the Neuronal Architecture and Cognitive Function in Adult Mice
}

\author{
Wai-Yu Li $i^{a}$ Yi-Chun Chang ${ }^{a} \quad$ L. Jyuhn-Hsiarn Lee ${ }^{d, e} \quad$ Li-Jen Lee $^{a-c}$ \\ ${ }^{a}$ Graduate Institute of Anatomy and Cell Biology, ${ }^{\mathrm{b}}$ Institute of Brain and Mind Sciences and ${ }^{\mathrm{C}}$ Neurobiology and \\ Cognitive Science Center, National Taiwan University, and ${ }^{\mathrm{d}}$ Department of Neurology and Environmental and \\ Occupational Medicine, National Taiwan University Hospital, Taipei, and e Division of Environmental Health and \\ Occupational Medicine, National Health Research Institutes, Zhunan,Taiwan
}

\section{Key Words}

Prenatal infection · Short-term memory · Dopamine neuron - Dentate gyrus - Medial prefrontal cortex . Dendrites

\begin{abstract}
Environmental factors such as prenatal infection are involved in the pathogenic processes of neurodevelopmental psychiatric disorders. In the present study, we administered a viral mimic, polyriboinosinic-polyribocytidylic acid (poly I:C, $20 \mathrm{mg} / \mathrm{kg}$, i.p.), to pregnant B6 mice at gestational day 9.5. Neonates born to these poly I:C-treated dams showed an increase of microglia in the hippocampus, indicating an activation of the immune system in the brains. Moreover, a significant increase in the number of dopamine-producing neurons in the ventral tegmental area was observed in adult male poly $1: C$ offspring compared with age-matched saline offspring. Poly I:C offspring also exhibited hypolocomotor activity in a novel open-field arena but did not display signs of anxiety or depression in the elevated plus maze or the forced swim test, respectively. However, the short-term memory of the poly I:C offspring was impaired in a novel object recognition task. Therefore, the dendritic architecture of granule cells in the dentate gyrus (DG) and pyramidal neurons in the medial prefrontal cortex (mPFC) were examined.
\end{abstract}

The dendritic complexity was reduced in the DG granule cells of the poly I:C offspring and exhibited shorter dendritic length compared with the saline offspring. The density of dendritic spines in the DG granule cells was also decreased in the poly I:C offspring. Furthermore, the dendritic complexity and spine density were reduced in layer II/III MPFC pyramidal neurons of the poly I:C offspring. Together, these data demonstrate impaired short-term memory and altered dendritic architecture in adult poly I:C offspring, which validates the prenatal infection paradigm as a model for neurodevelopmental psychiatric disorders.

(c) 2014 S. Karger AG, Basel

\section{Introduction}

The etiology of neurodevelopmental psychiatric disorders such as autism and schizophrenia involves disrupted brain development [1]. Adverse in utero events that influence the critical processes of brain development may predispose the emergence of mental diseases in adulthood [2]. Maternal infection has gained significant attention in recent years due to its association with various neurode-

Wai-Yu Li and Yi-Chun Chang contributed equally in this work.

\section{KARGER}

E-Mail karger@karger.com

www.karger.com/dne
(C) 2014 S. Karger AG, Basel

$0378-5866 / 14 / 0365-0359 \$ 39.50 / 0$
Li-Jen Lee

Graduate Institute of Anatomy and Cell Biology College of Medicine, National Taiwan University No. 1, Section 1, Ren-Ai Rd, Taipei 100 (Taiwan) E-Mail ljlee@ntu.edu.tw 
velopmental psychiatric disorders, including autism and schizophrenia [3-5]. Functional changes in neuropsychiatric diseases have been extensively studied; however, structural changes in the brain at cellular level were less addressed [6].

Polyriboinosinic-polyribocytidylic acid (poly I:C), which is a synthetic double-stranded RNA that stimulates viral infection-induced physiological reactions [7, 8], has been widely used as a viral mimic [9]. Administration of poly I:C to pregnant animals often leads to robust impacts on the offspring, indicating a transplacental effect of maternal immune activation on the developing fetus $[5,10]$.

The use of animals to model human-specific diseases, particularly neuropsychiatric disorders, has long been debated [11]. Some symptoms of schizophrenia, such as delusion and hallucination, cannot be diagnosed in animals. However, changes in locomotor activity, emotion-related behaviors and cognitive functions manifest in both animal models of schizophrenia and human patients [12]. Behavioral and structural phenotypes are therefore useful criteria for animal models of mental disorders. In addition, animal models provide in-depth examinations in the biochemical, physiological and histological aspects that can be assessed at any time point.

In the present study, we administered poly I:C to pregnant mice at gestational day 9.5. Behavioral, neurochemical and morphological phenotypes were characterized in the adult male offspring of these poly I:C-infected dams. Our findings demonstrate that the function and structure of the hippocampus and medial prefrontal cortex (mPFC) are impaired in poly I:C offspring, which validates the prenatal infection paradigm as an animal model of neurodevelopmental mental disorders.

\section{Materials and Methods}

\section{Animals}

Pregnant C57BL/6J mice obtained from the National Laboratory Animal Center were administered either a single injection of poly I:C (20 mg/kg, i.p.; Sigma, St. Louis, Mo., USA) or saline at gestational day 9.5, which corresponds approximately to the first trimester of a human pregnancy. The dose and timing of the poly I:C treatment were based on previous studies [13]. After weaning (postnatal day 30, PD30), the mice were group-housed (3-5) in the Laboratory Animal Center of the College of Medicine, National Taiwan University, under a 12:12 light-dark cycle with free access to food and water. Only male offspring were used in the present study. A series of behavioral tests were conducted after PD60, and followed by histological experiments. All animal manipulations were in accordance with a protocol approved by the Institutional Animal Care and Use Committee of the Colleges of
Medicine and Public Health, National Taiwan University. All efforts were made to minimize animal suffering and the number of animals used.

\section{Behavioral Tests}

Adult male offspring born to poly I:C- and saline-treated mothers (poly I:C and saline offspring, respectively) were subjected to behavioral tests. These tests were conducted during the light phase between 10 a.m. and 4 p.m. Prior to the start of each behavioral test, the mice were brought to the test place for habituation for at least $30 \mathrm{~min}$. Instruments were cleaned with $10 \%$ alcohol between tests. A total of 14 poly I:C offspring and 12 saline offspring from 9 litters were used.

Open-Field Test. After habituation, the mice were placed in a novel open-field arena $(40 \times 40 \mathrm{~cm})$ and allowed to explore freely for $10 \mathrm{~min}$. Their behavior was continuously recorded by a video camera placed above the arena. The floor of the arena was divided equally into 25 squares. The 9 central squares were denoted as the central area and the surrounding 16 squares were the peripheral area. The distances traveled and time spent within the central and peripheral areas were analyzed by TopScan LITE (Clever Systems, Reston, Va., USA).

Novel Object Recognition Test. Prior to the test, the mice were placed in an open-field arena for 10 min twice a day for 2 days for habituation. During the exploration phase, a mouse was placed in the open field and presented with a pair of identical objects for $8 \mathrm{~min}$. The mouse was then returned to its home cage for $10 \mathrm{~min}$ as a retention period. In the test phase, the mouse was returned to the open field and presented with one familiar object and one novel object. The mouse was again allowed to inspect the objects for $8 \mathrm{~min}$. The time spent in exploration behavior, such as deliberate contact or touching the object with the nose, was quantified. The discrimination ratio, (novel object exploration time/familiar object exploration time), was used to evaluate the performance of short-term recognition memory.

Elevated Plus Maze. The elevated plus maze was constructed with white acrylic boards and consisted of two crossed open arms $(40 \times 6 \mathrm{~cm})$ and two closed arms enclosed by $20-\mathrm{cm}$ high walls and located $50 \mathrm{~cm}$ above the floor. During the test, a mouse was placed on the central platform of the maze with its head facing one of the open arms and allowed to move freely for $10 \mathrm{~min}$. The behavior was recorded by a video camera and analyzed with TopScan LITE (Clever Systems). The distance traveled and time spent in the open arms, closed arms and central region were quantified.

Forced Swim Test. Each mouse was confined in a narrow Plexiglas cylinder ( $25 \mathrm{~cm}$ height, $10 \mathrm{~cm}$ internal diameter) filled with warm water $\left(25^{\circ} \mathrm{C}, 15 \mathrm{~cm}\right.$ height $)$. Three behavioral parameters, including struggling (mouse stretched forepaws out of the water to escape), immobility (mouse remained calmly in the water with its head above the water and maintained balanced for more than $2 \mathrm{~s}$ ) and swimming (mouse tried to keep its body from sinking by moving its hind paws), were quantified.

\section{Histology}

After the behavioral tests, at around PD80, the adult mice were sacrificed using an overdose of chloral hydrate (7\%) and transcardially perfused with phosphate-buffered saline (PBS) followed by a fixative (4\% paraformaldehyde in phosphate buffer, $\mathrm{pH} 7.4$ ). Some neonatal (PD2) mice were sacrificed and perfused in the same way. After perfusion, the brains were removed and stored in PBS-containing sodium azide $(0.1 \%)$. 
Immunohistochemistry and Stereological Examinations. Coronal sections of the brain were sectioned at a thickness of $50 \mu \mathrm{m}$ with a vibrating microtome. The sections were then reacted with $10 \%$ methanol and $0.3 \% \mathrm{H}_{2} \mathrm{O}_{2}$ in PBS for 10 min to block endogenous peroxidase activity. After PBS rinses, the sections were transferred to a blocking solution containing $4 \%$ normal goat serum, $1 \%$ bovine serum albumin and $0.4 \%$ Triton X-100 for $1 \mathrm{~h}$. After blocking, the sections were incubated with mouse anti-tyrosine hydroxylase (TH; 1: 2,000; Sigma) or rabbit anti-Iba1 (1:1,000; GeneTex, Hsinchu, Taiwan) overnight at $4^{\circ} \mathrm{C}$. After PBS washes, the sections were incubated with biotinylated second antibodies (1:500; Vector Laboratories, Burlingame, Calif., USA) and followed by avidin-biotin peroxidase complex (ABC kit; Vector Laboratories). Finally, the sections were reacted with $3,3^{\prime}$-diaminobenzidine (with $0.01 \%$ $\mathrm{H}_{2} \mathrm{O}_{2}$ in PBS) and mounted.

The density of Iba1-positive microglia in the hippocampus and $\mathrm{TH}$-positive neurons in the ventral tegmental area (VTA) were estimated unilaterally by stereological methods. In brief, the sections were examined on a light microscope (Olympus, Tokyo, Japan) with a computer-controlled motorized stage using an optical fractionator sampling protocol in the Stereo Investigator system (MBF Bioscience, Williston, Vt., USA).

Golgi-Cox Impregnation and Morphometric Analyses. Whole brains were immersed in the impregnation solution from the FD Rapid Golgi Stain kit (NeuroTechnologies, Ellicott City, Md., USA) at room temperature for 3 weeks. Impregnated samples were then cut at a thickness of $150 \mu \mathrm{m}$ using a vibratome. The sections were incubated with a mixture of developer and fixer solutions (FD Rapid Golgi Stain kit) and washed, mounted and examined under a light microscope with a $20 \times$ objective lens for dendritic morphology or a $100 \times$ objective lens for spine analysis. Series of pictures were taken with the Stereo Investigator system (MBF Bioscience). The morphology of selected neurons was reconstructed and analyzed with Neurolucida software (MBF Bioscience). The Sholl and branched structure analyses from the Neurolucida Explorer software toolbox were used to quantify topological parameters (e.g. numbers of primary dendrites, branching nodes, dendritic segments and terminal endings) and size-related parameters (e.g. dendritic length, intersections and terminal endings at different distances from the soma). The spine density was measured in dendritic segments with the aid of ImageJ software (NIH).

\section{Statistical Analysis}

Two-tailed unpaired Student's t tests were used for statistical analysis. To evaluate the interaction between treatments and time points, two-way ANOVA was performed and followed by t tests. Data were expressed as the mean \pm SEM. A value of $\mathrm{p}<0.05$ indicated statistical significance between the poly I:C and saline-treated offspring.

\section{Results}

The Number of Microglia Was Transiently Increased in Poly I:C Offspring

Subsequent to inflammation insult, cytokines produced by the maternal peripheral immune system may enter the fetus and impair the neurodevelopmental pro- cesses [10]. To examine the activity of the brain immune system of the offspring, the density of microglia was quantified. Microglial cells in the hippocampus were revealed by Iba1 immunohistochemistry using PD2 and PD80 mice (fig. 1a, b). A significant interaction between treatments and time points was noticed $(\mathrm{F}=21.596, \mathrm{p}<$ $0.0001)$. At PD2, the density of Iba1-positive microglia was greater in poly I:C offspring compared with saline offspring $(\mathrm{F}=26.411, \mathrm{p}<0.001)$. However, the density was comparable between the two groups at PD80 $(\mathrm{F}=$ $3.591, \mathrm{p}=0.075)$. These results suggest that the immune system of the poly I:C offspring was transiently activated in the brain during the perinatal period following maternal poly I:C exposure; however, this activation did not extend into adulthood. These results were consistent with the findings in a prenatal poly I:C exposure model using spiny mice [14].

\section{Increased TH-Positive Neurons in the VTA of Adult Poly I:C Offspring}

The mesocorticolimbic dopamine system plays an important role in emotion and cognitive function, and its development is affected by prenatal infection [15]. The density of dopamine-producing neurons in the VTA was estimated in the adult offspring of poly I:C and salinetreated dams. Dopamine neurons in the VTA were revealed by $\mathrm{TH}$ immunohistochemistry (fig. 2a) and quantified using an unbiased stereological method. A greater number of TH-positive neurons were observed in the VTA of adult male poly I:C offspring (fig. $2 \mathrm{~b}$ ) compared with age-matched saline offspring, which implies that the dopamine system was altered in the poly I:C offspring.

\section{Poly I:C Offspring Exhibited Hypolocomotor Activity without Signs of Anxiety or Depression}

The locomotor activity of the mice was examined in an open-field arena. Poly I:C offspring exhibited a reduction in the distance traveled compared with the saline offspring (fig. 3a). Reduced locomotor activity is also common in animal models of depression and anxiety [16]. The level of anxiety was estimated by the time spent in the open arm of the elevated plus maze. Among the two groups, the time spent in the open arms and other regions were comparable (fig. 3b). The degree of depression was evaluated by the duration of immobility in the forced swim test. Both the poly I:C and saline offspring spent the same amount of time in a state of immobility as well as struggling and swimming (fig. 3c). Together, these results indicate that the emotional status of adult offspring was not affected by maternal poly I:C exposure. 


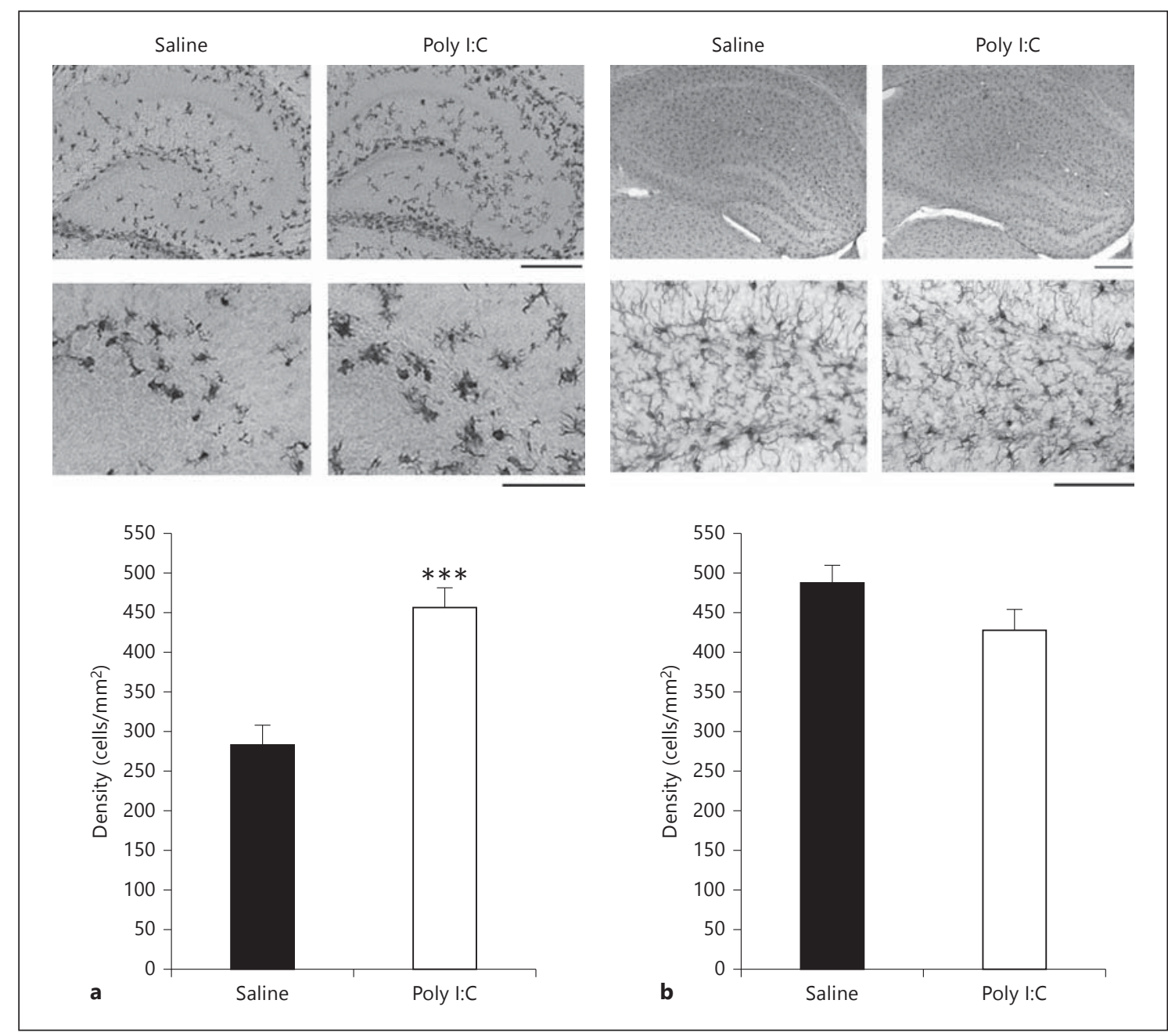

Fig. 1. Numbers of microglia in the hippocampus of neonatal and adult mice. Microglia in the hippocampus were revealed by Iba1 immunohistochemistry in PD2 (a) and PD80 (b) mice. A higher density of Iba1-positive cells was observed in poly I:C offspring at
PD2 but not at PD80. The bars in $\mathbf{a}$ and $\mathbf{b}$ indicate $100 \mu \mathrm{m}$. Asterisks indicate a significant difference between the saline $(\mathrm{n}=3$ mice $)$ and poly I:C ( $\mathrm{n}=4$ mice $)$ offspring. ${ }^{* * *} \mathrm{p}<0.001$.

\section{Impaired Short-Term Memory in Adult Poly I:C Offspring}

The effects of maternal poly I:C administration on the cognitive function of adult offspring were evaluated by a novel object recognition test. During the exploration phase, the discrimination ratio was close to 1 in both groups, indicating the absence of location preference of the objects. During the test phase, the saline offspring spent more time exploring the novel object than the familiar one, resulting in a high discrimination ratio $(>1.5)$, which indicates that the saline offspring retained shortterm memory-based discrimination between novel and familiar objects. However, in poly I:C offspring, the time spent in examining the novel and familiar objects was al- most the same (discrimination ratio approx. 1; fig. 4b), indicating that the short-term memory of the poly I:C offspring was impaired.

\section{Dendritic Structures of Granule Cells in the DG Were}

\section{Changed in Poly I:C Offspring}

Adult poly I:C offspring exhibited impaired recognition memory function, which is largely mediated by the functions of the hippocampus and $\mathrm{mPFC}$ [17]. Therefore, the granule cells in the dentate gyrus (DG), which is important for recognition memory [18], were analyzed. The dendritic structure of the DG granule cells in the dorsal hippocampus was reconstructed (fig. $5 \mathrm{a}-\mathrm{c}$ ). Some granule cells had a single primary dendrite (fig. 5b) while oth- 

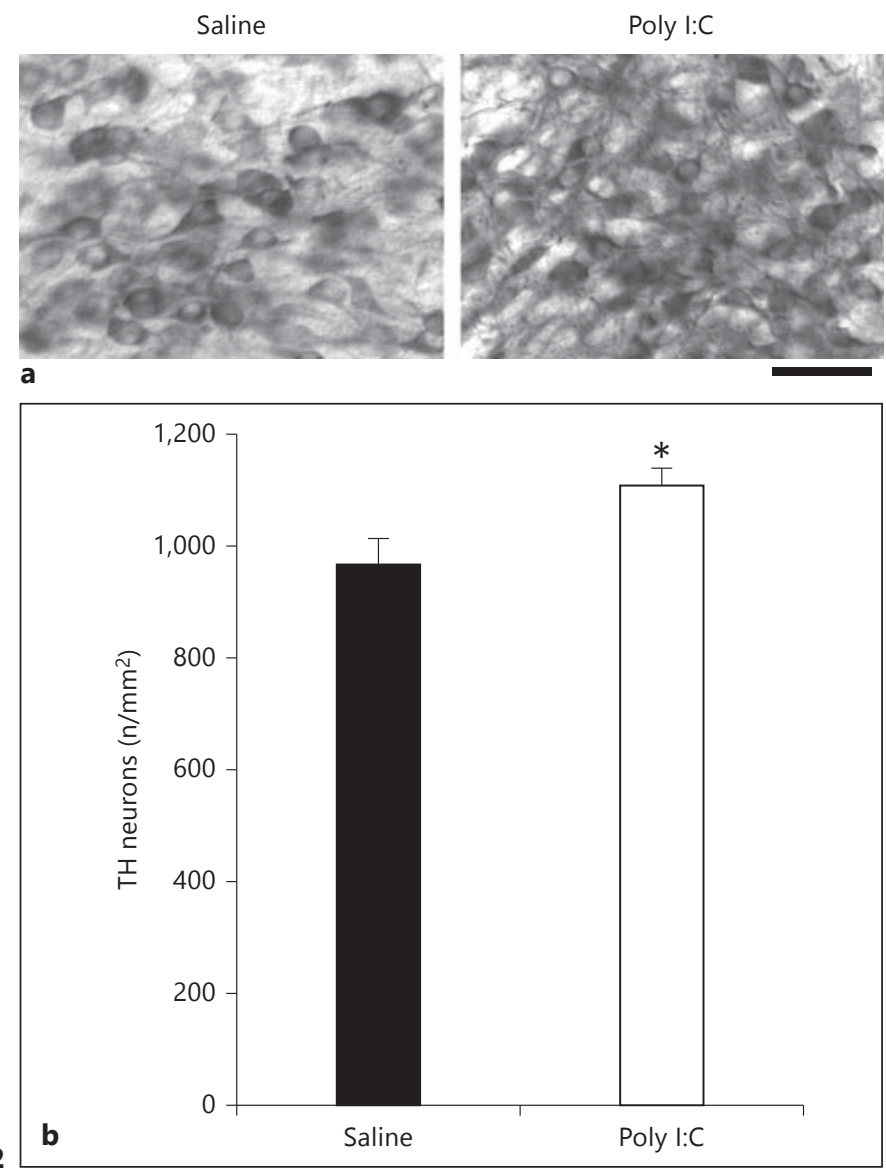

2

Fig. 2. TH-positive neurons in the VTA. a Coronal sections containing the VTA were stained with anti-TH antibody; scale bar = $50 \mathrm{~mm}$. b The density of TH-positive neurons was increased in the poly I:C group. Results are mean \pm SEM. Asterisks indicate a significant difference between the saline $(\mathrm{n}=5$ mice $)$ and poly I:C ( $\mathrm{n}=6$ mice) groups. ${ }^{*} \mathrm{p}<0.05$.

Fig. 3. Adult poly I:C offspring exhibit hypolocomotor activity without changes in anxiety and depression levels. a Locomotor activity was measured in a novel open-field arena for $10 \mathrm{~min}$. The total distance traveled by the poly I:C offspring was decreased compared with saline-offspring. b In the elevated plus maze test, poly I:C offspring spent a similar amount of time in the open arm as the saline offspring, indicating a comparable anxiety level between groups. c In the forced swim test, there were no significant differences in the time spent in each behavior between the poly I:C and saline-offspring, indicating similar depression levels. Results are mean \pm SEM. Asterisks indicate significant differences between the saline $\left(\mathrm{n}=11\right.$ mice) and poly I:C $\left(\mathrm{n}=14\right.$ mice) groups. ${ }^{*} \mathrm{p}<0.05$.

ers had multiple primary dendrites (fig. 5c). Multipledendrite DG granule cells are usually mature cells, whereas single-dendrite granule cells include immature cells [19]. The dendritic profile was evaluated using the concentric-ring method of Sholl. In the poly I:C offspring, the numbers of intersections were reduced in both single-

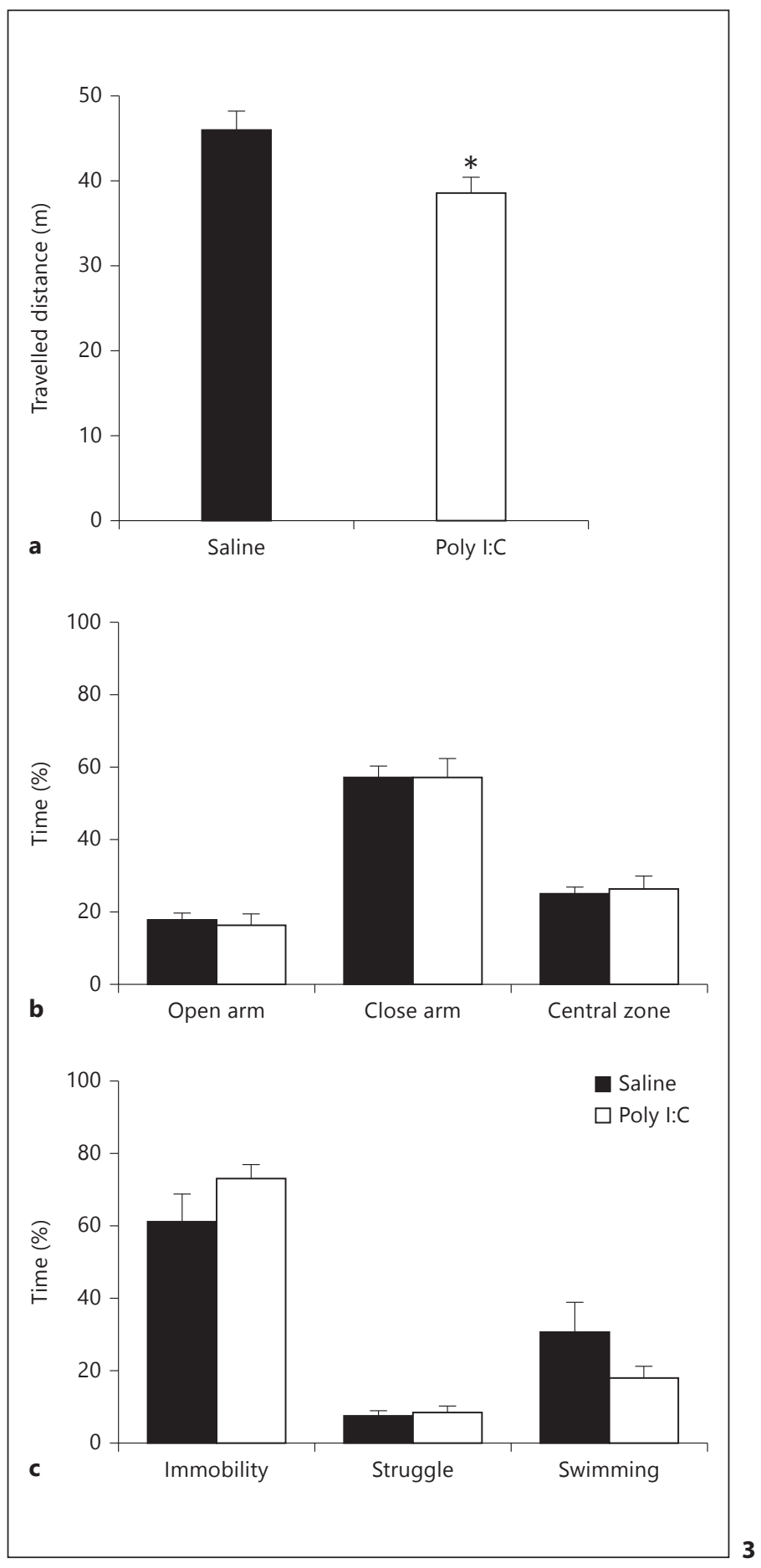

and multiple-dendrite granule cells compared with the saline offspring (fig. 5d, e). Furthermore, the total dendritic length was reduced in the granule cells of the poly I:C offspring (table 1). The numbers of segments, bifurcation nodes and terminal endings were changed in the multiple-dendrite granule cells of the poly I:C offspring 


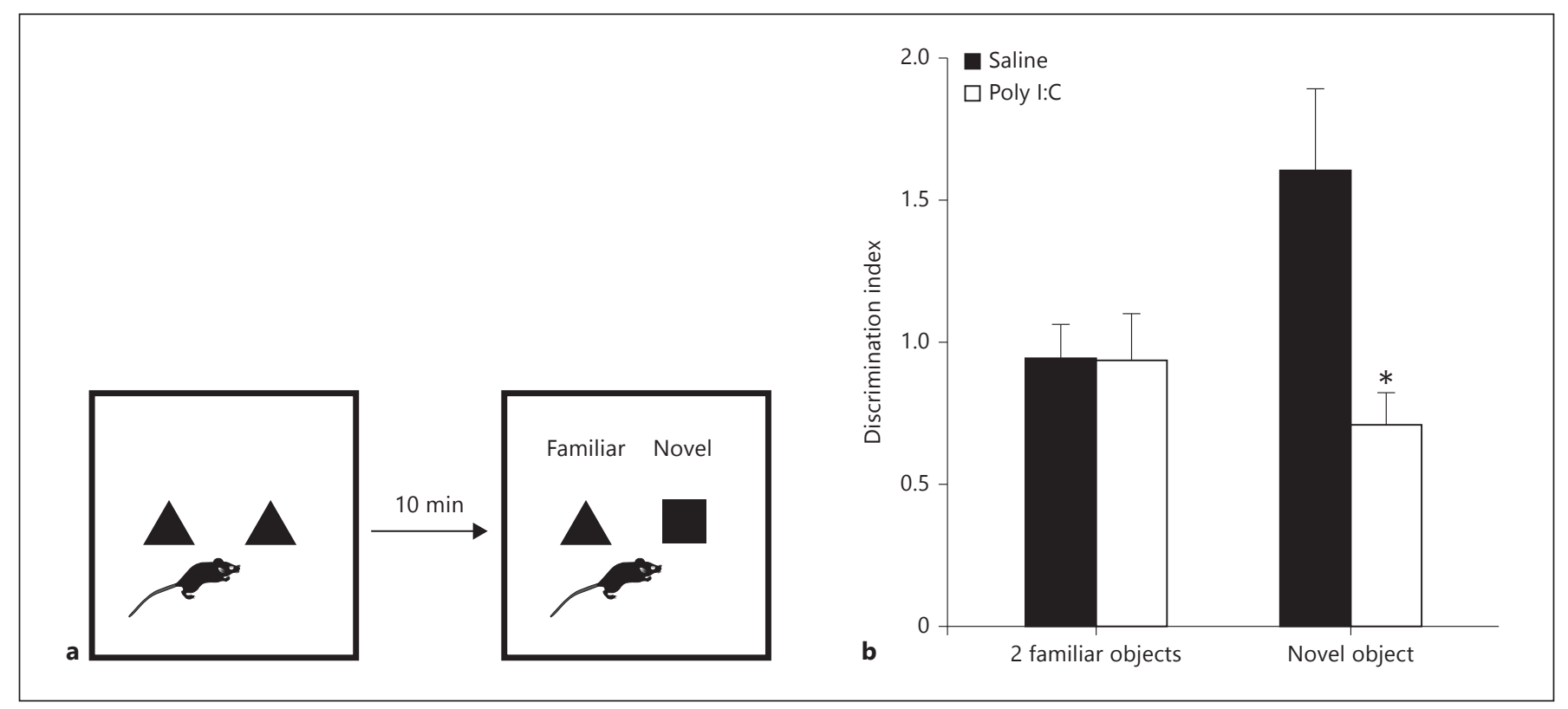

Fig. 4. Short-term memory on the novel object recognition task. a A schematic representation of the novel object recognition task. During the exploration phase, the discrimination ratio was close to 1 in both groups, indicating a lack of location preference of the objects (left in b). In the test phase, saline offspring spent a greater amount of time exploring the novel object compared with the poly
I:C offspring in which the discrimination ratio was about 1 (right in b). These results suggest that the poly I:C offspring had impaired object recognition memory. Results are mean \pm SEM. Asterisks indicate significant differences between the saline ( $\mathrm{n}=6$ mice) and poly I:C ( $\mathrm{n}=6$ mice) groups. ${ }^{*} \mathrm{p}<0.05$.

Table 1. Morphometric features of DG granule cells

\begin{tabular}{lcclcc}
\hline Parameters & \multicolumn{2}{l}{ Single-dendrite cells } & & \multicolumn{2}{l}{ Multiple-dendrite cells } \\
\cline { 2 - 3 } \cline { 5 - 6 } & saline $(\mathrm{n}=18)$ & poly I:C $(\mathrm{n}=18)$ & & saline $(\mathrm{n}=14)$ & poly I:C $(\mathrm{n}=15)$ \\
\hline Primary dendrites & $1 \pm 0$ & $1 \pm 0$ & & $2.79 \pm 0.26$ & $2.13 \pm 0.09^{*}$ \\
Bifurcation nodes & $6.89 \pm 0.4$ & $6.06 \pm 0.39$ & & $7.36 \pm 0.63$ & $5.47 \pm 0.36^{*}$ \\
Terminal endings & $8.33 \pm 0.46$ & $7.56 \pm 0.37$ & & $10.5 \pm 0.63$ & $7.73 \pm 0.41^{* * *}$ \\
Highest order & $5.28 \pm 0.29$ & $4.89 \pm 0.21$ & & $4.07 \pm 0.3$ & $3.93 \pm 0.25$ \\
Total dendritic length, $\mathrm{m}$ & $1,021.1 \pm 35.03$ & $757.02 \pm 53.38^{* * *}$ & & $1,129.6 \pm 60.96$ & $797.99 \pm 63.94^{* * *}$ \\
\hline
\end{tabular}

Results were collected from 6 saline- and 7 poly I:C-exposed mice. n: number of neurons. Data are reported as the mean \pm SEM. ${ }^{*} \mathrm{p}<0.05 ;{ }^{* * *} \mathrm{p}<0.001$.

(fig. 5f, g; table 1). The lengths of the dendritic segments, which can be classified as either intermodal (between two bifurcation nodes) or terminal, were quantified. The length of the terminal segments was reduced in the poly I:C offspring, especially in single-dendrite granule cells. However, the intermodal length was reduced in multipledendrite granule cells of the poly I:C offspring (fig. 5h). We then examined the density of the dendritic spines in the DG granule cells. Fewer dendritic spines were found in the DG granule cells of the poly I:C offspring (fig. $5 \mathrm{i}$ ).

\section{Dendritic Architecture of Layer II/III mPFC Pyramidal Neurons}

Layer II/III pyramidal neurons in the mPFC from saline and poly I:C offspring were examined. Only the basilar dendrites were analyzed because the apical dendrites were scarce (fig. 6; table 2). The dendritic profile was evaluated using the concentric-ring method of Sholl. Compared with the saline group, the numbers of intersections in the poly I:C group were reduced in the proximal regions $(<50 \mu \mathrm{m}$ from the soma) but increased in the more 


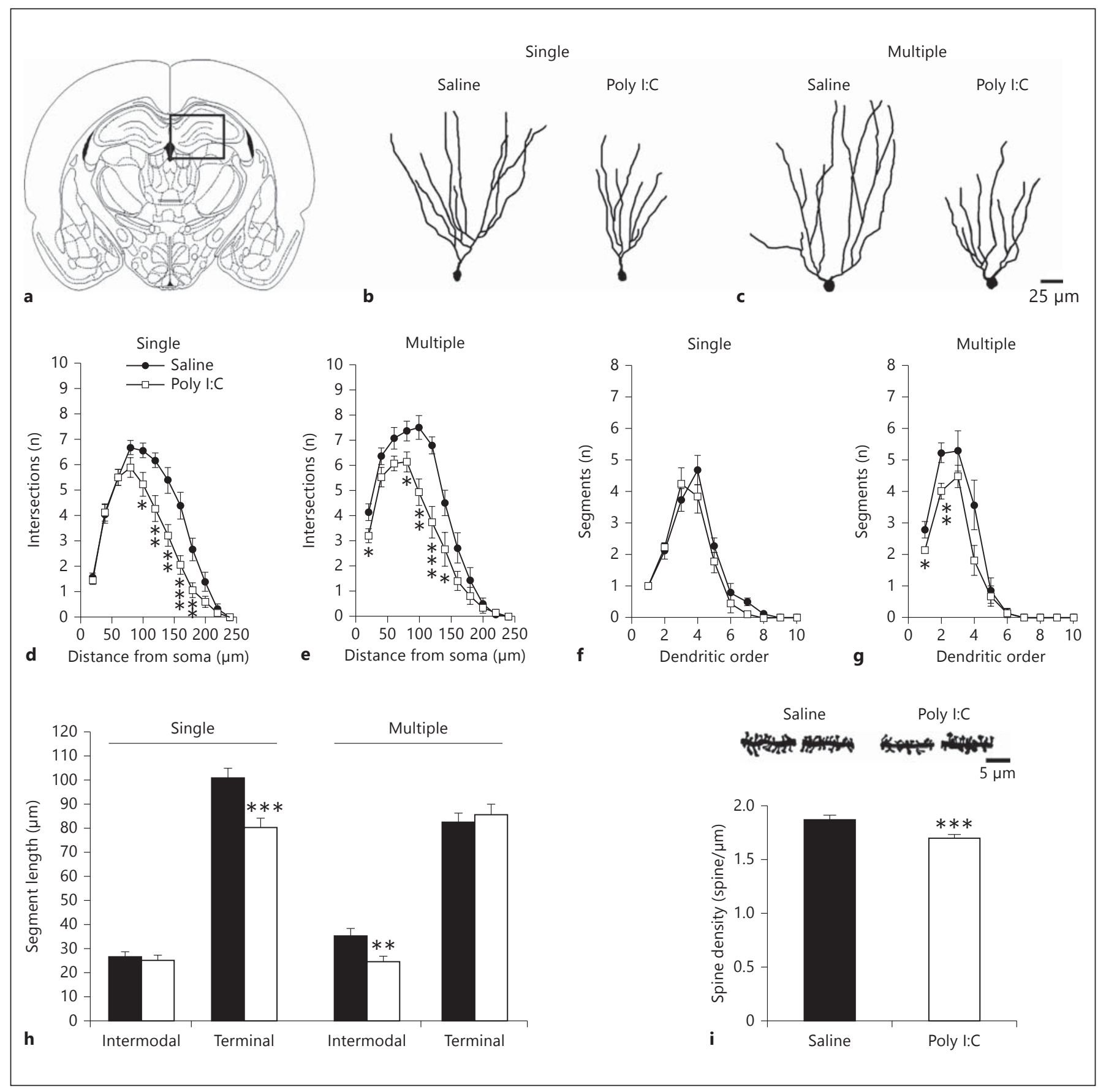

Fig. 5. Dendritic structures of the DG granule cells. a The dorsal hippocampus is indicated by a square in the coronal section of the mouse brain. Examples of reconstructed Golgi-Cox impregnated granule cells with a single primary dendrite (b) or multiple dendrites (c) are illustrated. $\mathbf{d - g}$ For quantitative comparisons, 18 single-dendrite and 14 multiple-dendrite DG granule cells were collected from 6 saline offspring; 18 single-dendrite and 15 multipledendrite DG granule cells were collected from 7 poly I:C offspring. d, e The complexity of dendritic arbors was evaluated with the concentric method of Sholl. In the poly I:C offspring, the numbers of intersections were reduced in single-dendrite and multiple-den- drite granule cells compared with the saline offspring. The number of segments was comparable in single-dendrite granule cells among poly I:C and saline offspring (f), whereas it was reduced in multiple-dendrite granule cells in poly I:C offspring compared with saline-treated offspring ( $(\mathbf{g})$. $\mathbf{h}$ In the poly I:C offspring, shorter dendritic segment was noticed in the terminal segments of single-dendrite granule cells and intermodal segments of multipledendrite granule cells. i Examples of dendritic segments of DG granule cells: fewer dendritic spines were observed in poly I:C offspring. Results are mean \pm SEM. Asterisks indicate significant differences between groups. ${ }^{*} \mathrm{p}<0.05$; ${ }^{* *} \mathrm{p}<0.01$; ${ }^{* *} \mathrm{p}<0.001$. 
distal regions ( $>100 \mu \mathrm{m}$ from the soma; fig. $6 \mathrm{c})$. This probably resulted from the longer segment length in both the intermodal and terminal segments (fig. 6d, e). However, the total numbers of primary dendrites, bifurcation nodes, terminal endings and dendritic segments as well as the total dendritic length of the basilar dendrites in layer II/III mPFC neurons were not significantly altered in the poly I:C offspring (table 2).

The density of the dendritic spines in the mPFC neurons was examined. The spine density was reduced in the proximal regions $(<100 \mu \mathrm{m}$ from the soma) of the apical dendrites (fig. 6f), including the apical trunk and collaterals, in neurons of the poly I:C group. In the basilar dendrites, reduced spine density was also evident in neurons from the poly I:C offspring (fig. $6 \mathrm{~g}$ ). Together, these changes may be the anatomical basis for the impairment in $\mathrm{mPFC}$-associated functions in the poly I:C offspring.

\section{Discussion}

In this study, we administered poly I:C to pregnant mice at gestational day 9.5, which is equivalent to the first trimester in human gestation. We observed brain and behavioral changes in the offspring born to poly I:Ctreated mothers, including a transient increase in the microglia in the hippocampus, a greater number of dopamine-producing cells in the VTA, impaired short-term object recognition memory and altered dendritic architecture in both the DG granule cells and layer II/III pyramidal $\mathrm{mPFC}$ neurons. These structural changes may contribute to the deficit in cognitive function observed in these animals.

\section{Implications of Increased Microglia in the Poly I:C Offspring}

In the present study, more Iba1-positive microglial cells were observed in the hippocampus of neonatal (PD2) poly I:C offspring compared with the saline off-

Fig. 6. Dendritic arborization in the layer II/III pyramidal neurons of the mPFC. a The location of the mPFC is marked in gray in coronal sections of the mouse brain. $\mathbf{b}$ Examples of Golgi-Cox impregnated layer II/III pyramidal neurons in the mPFC are illustrated. Apical dendrites were omitted in these cases; $15 \mathrm{mPFC}$ neurons collected from 6 saline offspring and $14 \mathrm{mPFC}$ neurons collected from 7 poly I:C offspring were used for quantitative comparisons. c The numbers of intersections were counted in relation to the distance from the soma center. d, e Lengths of intermodal and terminal dendritic segments were measured in relation to the dendritic order. $\mathbf{f}$ Dendritic fragments collected from the trunk spring. However, this increase did not extend into adulthood, indicating a transient activation of the immune system in the brain during the perinatal stages. Microglia are immunocompetent cells in the brain that are involved in the processes of brain injury and inflammation. They are derived from a distinct population of myeloid precursor cells that invade the brain on gestational day 9.5 in mice [20]. A higher number of hippocampal microglia in the neonatal poly I:C offspring suggests that the migration of myeloid precursor cells into the brain is promoted by an early immune challenge because the poly I: $\mathrm{C}$ was administered at gestational day 9.5. A greater number of hippocampal microglia has been observed in neonates born to lipopolysaccharide (LPS)-infected mothers [21], which indicates that maternal LPS exposure induced inflammation in the fetal brain. Microglia are activated by inflammatory cytokines such as interleukin-6 (IL-6), which is elevated in both the mother and fetus following prenatal LPS treatment [10]. Because IL-6 is a placenta-permeable inflammatory cytokine $[22,23]$, a dynamic inflammatory process in the fetal brain may therefore be elicited when pregnant mothers are exposed to infectious agents. Our study provides evidence to support a transient inflammatory reaction that features increased microglia in the brain of poly I:C offspring during the perinatal period. Inflammation in the fetal or neonatal brain can affect subsequent brain development and function that may be implicated in neurodevelopmental diseases [24].

\section{The Mesocorticolimbic Dopamine System Is Affected by Prenatal Poly I:C Exposure}

The mesocorticolimbic dopamine system includes the VTA, in which dopamine-producing neurons are located, and regions that receive dopaminergic projections, such as the amygdala, nucleus accumbens, prefrontal cortex and hippocampus. This system profoundly impacts various brain functions, including emotion and cognition

and collaterals from the proximal $(<100 \mu \mathrm{m}$ from soma) and distal $(<50 \mu \mathrm{m}$ from surface) regions of the apical dendrites were used for spine analysis. $\mathrm{Tp}=$ Trunk proximal; $\mathrm{Cp}=$ collateral proximal; $\mathrm{Td}=$ trunk distal; $\mathrm{Cd}=$ collateral distal. $\mathbf{g}$ For the basilar dendrites, fragments from different dendritic orders were collected. Reduced spine density was evident in both apical and basilar dendrites in the poly I:C group compared with the saline-treated group. Results are reported as the mean \pm SEM. Asterisks indicate significant differences between the saline-treated and poly I:C groups. ${ }^{*} \mathrm{p}<0.05$; ${ }^{* *} \mathrm{p}<0.01 ;{ }^{* * *} \mathrm{p}<0.001$.

(For figure 6 see next page.) 


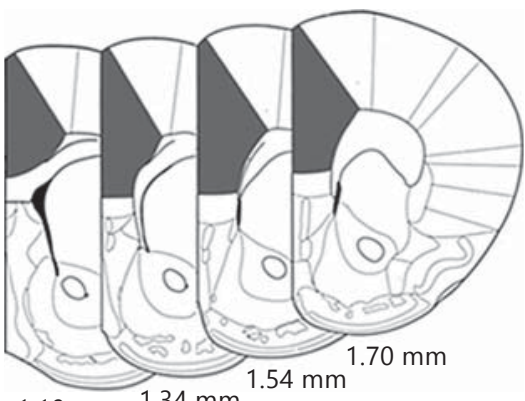

$1.10 \mathrm{~mm} 1.34 \mathrm{~mm}$

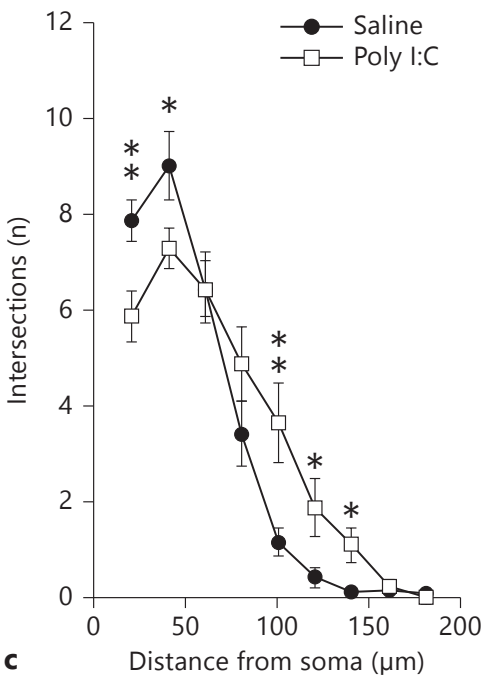

Apical dendrites

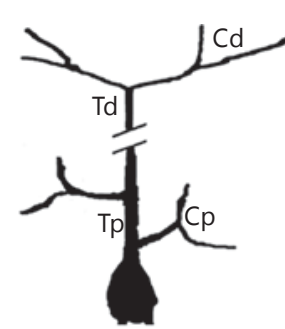

Saline Poly I:C

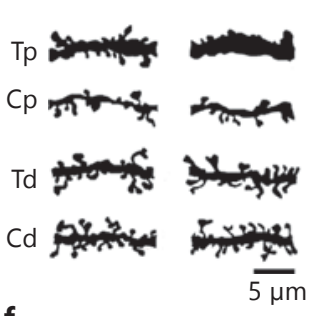

f

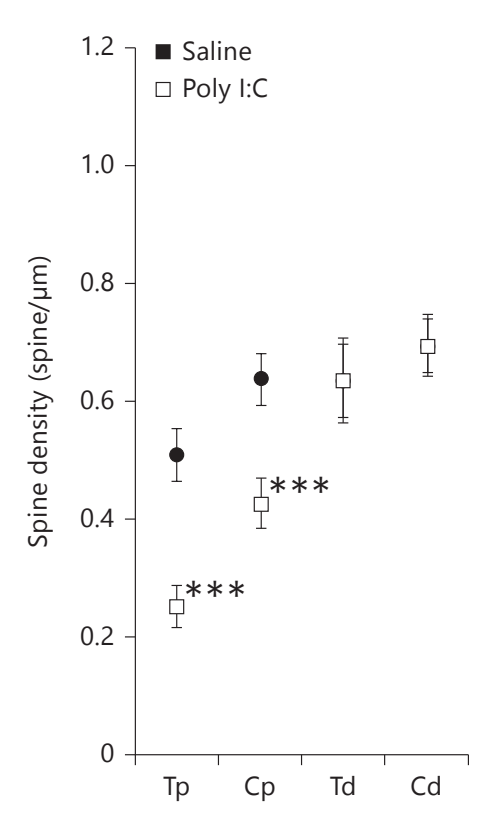

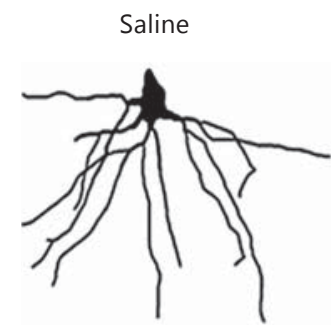

b

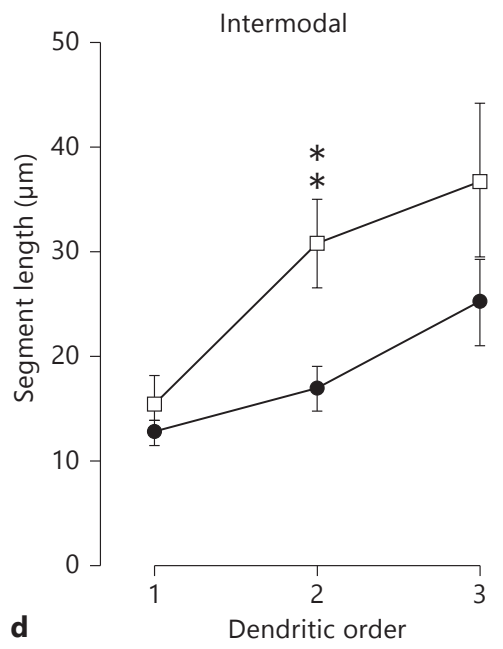

Basilar dendrites
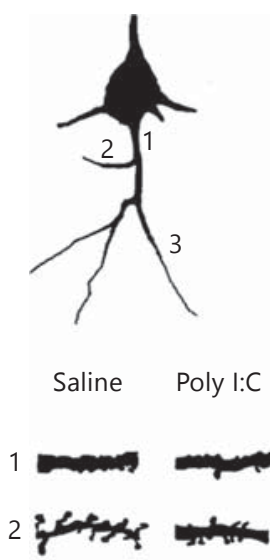

$3 \sin 3404$

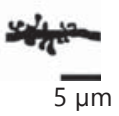

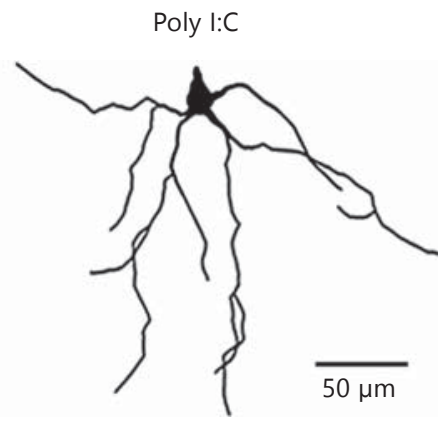
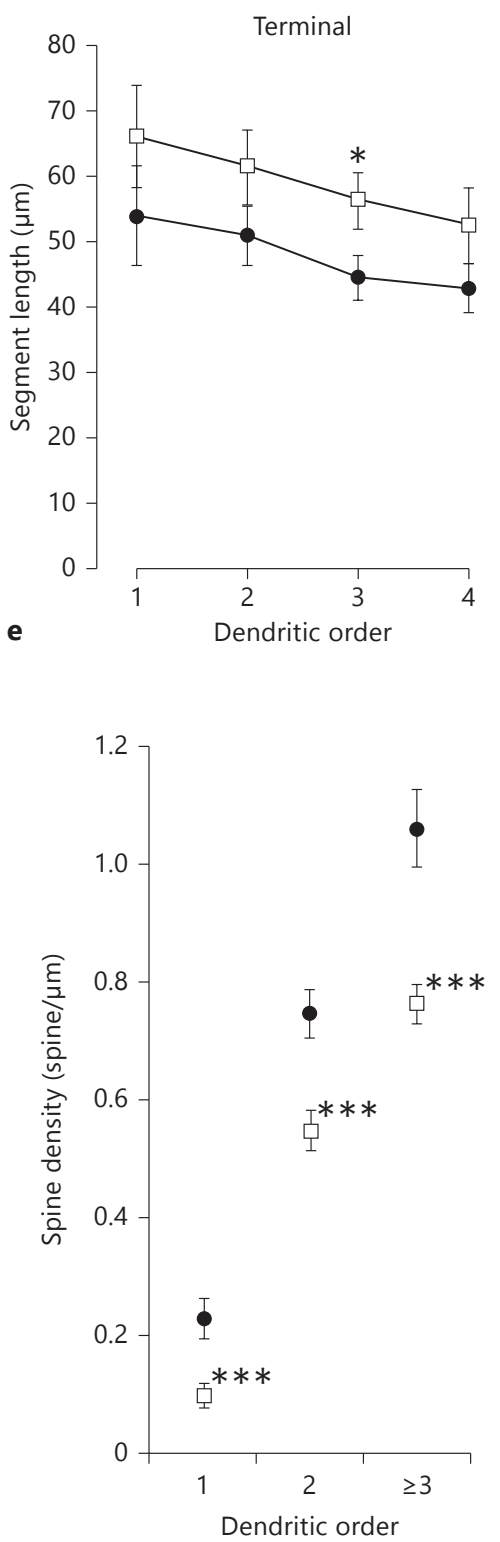
Table 2. Morphometric features of basilar dendrites in layer II/III pyramidal neurons in the $\mathrm{mPFC}$

\begin{tabular}{lcc}
\hline Parameters & Saline $(\mathrm{n}=15)$ & Poly I:C $(\mathrm{n}=14)$ \\
\hline Primary dendrites & $4.67 \pm 0.19$ & $4.29 \pm 0.34$ \\
Bifurcation nodes & $7.2 \pm 0.36$ & $6.07 \pm 0.66$ \\
Terminal endings & $12.4 \pm 0.51$ & $10.64 \pm 0.78$ \\
Segments & $19.6 \pm 0.84$ & $16.71 \pm 1.39$ \\
Highest order & $3.8 \pm 0.15$ & $3.64 \pm 0.20$ \\
Total dendritic length, $\mathrm{m}$ & $705.05 \pm 44.24$ & $777.15 \pm 65.80$ \\
\hline
\end{tabular}

Results were collected from 6 saline- and 7 poly I:C-exposed mice. $n$ : number of neurons. Data are reported as the mean \pm SEM.

[25]. Dysregulation of dopamine-related function may be attributed to some neuropsychiatric disorders, such as schizophrenia [15]. The present study observed an increase in the number of TH-positive dopamine neurons in the VTA of the poly I:C offspring, which is in line with a previous study [26] and supports the hyper-dopamine hypothesis of schizophrenia [15].

Both the mPFC and hippocampus are mesocorticolimbic structures that receive dopaminergic projections and play important roles in object recognition memory [27-29]. Impaired object recognition memory function has been replicated in various models of prenatal infection $[14,30,31]$. In the present study, we demonstrated morphological alterations in DG granule cells and layer II/III mPFC pyramidal neurons in the poly I:C offspring. These structural changes may account for the functional deficits of the $\mathrm{MPFC}$ and hippocampus in these offspring [17].

New granule cells are continuously generated in the adult DG of the hippocampus. Impaired hippocampal neurogenesis has been shown in several prenatal infection models [32] and may partially explain the behavioral deficits in tests of object recognition $[18,33]$. Morphological changes in the DG granule cells of poly I:C offspring may also contribute to hippocampus-related functional defects. Dendritic arbors and spines are important structures in the collection and integration of neural signals. As the gate or filter of the hippocampal circuit, DG granule cells control the activity of the hippocampal neural network [34]. Reduced dendritic complexity and spine density in DG granule cells may compromise synaptic transmission and information processes, which may impair hippocampus-related cognitive functions. However, we also observed cell shape-specific changes in the DG of the poly I:C offspring. Reductions in dendritic complexity, total dendritic length and spine density were evident, especially in multiple-dendrite granule cells. These are usually mature cells, whereas single-dendrite granule cells include immature cells [19]. Our data therefore suggest that the maturation processes of DG granule cells may also be affected by prenatal infection.

The altered $\mathrm{mPFC}$ neuronal structure in the poly I:C offspring may also be associated with impaired cognitive functions. In patients with schizophrenia, reduced dendritic complexity and spine density in layer III PFC pyramidal neurons have been attributed to deficits in working memory $[35,36]$. Impaired dendritic architecture has been replicated in the MPFC neurons of animal models of schizophrenia [6,37]. Together, our results demonstrate an anatomical basis for the functional deficits that are induced by prenatal poly I:C exposure.

\section{Altered Dendritic Architecture in Poly I:C Offspring}

Reduced dendritic complexity and spine density were observed in the neurons of mesocorticolimbic structures, such as the DG and mPFC, of adult male poly I:C offspring. The underlying mechanisms were not addressed in our present study; however, poly I:C-induced dopamine hyperfunction and the elevation of inflammatory cytokines, as well as disrupted neurotransmission and gene expression in the fetal brain [26, 38-40], may provide some clues to the structural malformation. In cultured cortical neurons, dose-dependent dopamine neurotoxicity has been demonstrated [41], which implies a negative impact of excess dopamine on cortical neuron development. In vitro studies have also demonstrated the suppressive effects of inflammatory cytokines such as IL- 6 and TNF on the outgrowth and branching of cortical and hippocampal neurons [42, 43]. Besides, the glutamatergic neurotransmission system is also affected by prenatal poly I:C exposure. Ionotropic glutamate receptors, such as NMDA and AMPA receptors, are reduced in poly I:C offspring [44, 45]. Given that glutamatergic excitatory neurotransmission also plays a critical role in dendritic development [46], altered dendritic profile in our poly I:C offspring might be attributed to the dysfunction of glutamatergic neurotransmission. However, further validation of this possibility is required.

A recent microarray-based study revealed changes in the gene expression profile of fetal brains exposed to a maternal poly I:C injection. In addition to genes that are involved in glutamatergic neurotransmission, alterations in Disc1 and Robol expression were also observed [45]. 
Robo1 interacts with Slit1 to regulate the dendritic branching of cortical neurons [47]. Disc1 proteins play an important role in various stages of neural development $[1,48]$. Altered dendritic architecture has been observed in the MPFC and DG neurons of Disc1 knockout mice $[37,49]$. Together, these results suggest that maternal poly I:C exposure may induce numerous neurochemical changes that affect the developing neurons in the fetal brain and lead to abnormal behaviors in adult poly I:C offspring.

\section{Acknowledgments}

This work was supported by the National Science Council of the Republic of China (grant NSC 102-2628-B-002-014-MY3). We thank the staff of the imaging core at the First Core Labs, National Taiwan University College of Medicine, for technical assistance.

\section{Disclosure Statement}

The authors have no conflicts of interest to disclose.

\section{References}

$\checkmark 1$ Brandon NJ, Sawa A: Linking neurodevelopmental and synaptic theories of mental illness through DISC1. Nat Rev Neurosci 2011;12: 707-722.

-2 Brown AS: Prenatal infection as a risk factor for schizophrenia. Schizophr Bull 2006;32: 200-202.

-3 Meyer U, Feldon J, Dammann O: Schizophrenia and autism: both shared and disorderspecific pathogenesis via perinatal inflammation? Pediatr Res 2011;69:26R-33R.

-4 Brown AS, Patterson PH: Maternal infection and schizophrenia: implications for prevention. Schizophr Bull 2011;37:284-290.

5 Meyer U, Feldon J: To poly(I:C) or not to poly(I:C): advancing preclinical schizophrenia research through the use of prenatal immune activation models. Neuropharmacology 2012;62:1308-1321.

6 Baharnoori M, Brake WG, Srivastava LK: Prenatal immune challenge induces developmental changes in the morphology of pyramidal neurons of the prefrontal cortex and hippocampus in rats. Schizophr Res 2009;107:99-109.

-7 Kimura-Takeuchi M, Majde JA, Toth LA, Krueger JM: The role of double-stranded RNA in induction of the acute-phase response in an abortive influenza virus infection model. J Infect Dis 1992;166:1266-1275.

-8 Alexopoulou L, Holt AC, Medzhitov R, Flavell RA: Recognition of double-stranded RNA and activation of NF- $\mathrm{KB}$ by toll-like receptor 3. Nature 2011;413:732-738.

9 Majde JA: Viral double-stranded RNA, cytokines, and the flu. J Interferon Cytokine Res 2000;20:259-272.

10 Boksa P: Effects of prenatal infection on brain development and behavior: a review of findings from animal models. Brain Behav Immun 2010;24:881-897.

11 Nestler EJ, Hyman SE: Animal models of neuropsychiatric disorders. Nat Neurosci 2010; 13:1161-1169.

12 Jaaro-Peled H, Ayhan Y, Pletnikov MV, Sawa A: Review of pathological hallmarks of schizophrenia: comparison of genetic models with patients and nongenetic models. Schizophr Bull 2010;36:301-313.
13 Soumiya H, Fukumitsu H, Furukawa S: Prenatal immune challenge compromises the normal course of neurogenesis during development of the mouse cerebral cortex. J Neurosci Res 2011;89:1575-1585.

14 Ratnayake U, Quinn TA, Castillo-Melendez M, Dickinson H, Walker DW: Behaviour and hippocampus-specific changes in spiny mouse neonates after treatment of the mother with the viral-mimetic Poly I:C at mid-pregnancy. Brain Behav Immun 2012;26:1288-1299.

15 Meyer U, Feldon J: Prenatal exposure to infection: a primary mechanism for abnormal dopaminergic development in schizophrenia. Psychopharmacology 2009;206:587-602.

16 Bourin M, Petit-Demoulière B, Dhonnchadha BN, Hascöet M: Animal models of anxiety in mice. Fundam Clin Pharmacol 2007;21: 567-574.

17 Dickerson DD, Wolff AR, Bilkey DK: Abnormal long-range neural synchrony in a maternal immune activation animal model of schizophrenia. J Neurosci 2010;30:12424-12431.

18 Jessberger S, Clark RE, Broadbent NJ, Clemenson GD Jr, Consiglio A, Lie DC, Squire LR, Gage FH: Dentate gyrus-specific knockdown of adult neurogenesis impairs spatial and object recognition memory in adult rats. Learn Mem 2009;16:147-154.

19 Ambrogini P, Lattanzi D, Ciuffoli S, Agostini D, Bertini L, Stocchi V, Santi S, Cuppini R: Morpho-functional characterization of neuronal cells at different stages of maturation in granule cell layer of adult rat dentate gyrus. Brain Res 2004;1017:21-31.

20 Ginhoux F, Greter M, Leboeuf M, Nandi S, See P, Gokhan S, Mehler MF, Conway SJ, Ng LG, Stanley ER, Samokhvalov IM, Merad M: Fate mapping analysis reveals that adult microglia derive from primitive macrophages. Science 2010;330:841-845.

21 Roumier A, Pascual O, Béchade C, Wakselman S, Poncer JC, Réal E, Triller A, Bessis A: Prenatal activation of microglia induces delayed impairment of glutamatergic synaptic function. PLoS One 2008; 3:e2595.

22 Zaretsky MV, Alexander JM, Byrd W, Bawdon RE: Transfer of inflammatory cytokines across the placenta. Obstet Gynecol 2004;103: 546-550.

23 Dahlgren J, Samuelsson AM, Jansson T, Holmäng A: Interleukin-6 in the maternal circulation reaches the rat fetus in mid-gestation. Pediatr Res 2006;60:147-151.

24 Hagberg H, Gressens P, Mallard C: Inflammation during fetal and neonatal life: implications for neurologic and neuropsychiatric disease in children and adults. Ann Neurol 2012;71:444-457.

25 Laviolette SR: Dopamine modulation of emotional processing in cortical and subcortical neural circuits: evidence for a final common pathway in schizophrenia? Schizophr Bull 2007;33:971-981.

26 Vuillermot S, Weber L, Feldon J, Meyer U: A longitudinal examination of the neurodevelopmental impact of prenatal immune activation in mice reveals primary defects in dopaminergic development relevant to schizophrenia. J Neurosci 2010;30:1270-1287.

27 Broadbent NJ, Gaskin S, Squire LR, Clark RE: Object recognition memory and the rodent hippocampus. Learn Mem 2009; 17:5-11.

28 Weible AP, Rowland DC, Pang R, Kentros C: Neural correlates of novel object and novel location recognition behavior in the mouse anterior cingulate cortex. J Neurophysiol 2009; 102:2055-2068.

29 Chao OY, Pum ME, Huston JP: The interaction between the dopaminergic forebrain projections and the medial prefrontal cortex is critical for memory of objects: implications for Parkinson's disease. Exp Neurol 2013;247:373-382.

30 Ozawa K, Hashimoto K, Kishimoto T, Shimizu E, Ishikura $\mathrm{H}$, Iyo M: Immune activation during pregnancy in mice leads to dopaminergic hyperfunction and cognitive impairment in the offspring: a neurodevelopmental animal model of schizophrenia. Biol Psychiatry 2006; 59:546-554.

31 Coyle P, Tran N, Fung JN, Summers BL, Rofe AM: Maternal dietary zinc supplementation prevents aberrant behaviour in an object recognition task in mice offspring exposed to LPS in early pregnancy. Behav Brain Res 2009; 197:210-218. 
-32 Cui K, Ashdown H, Luheshi GN, Boksa P: Effects of prenatal immune activation on hippocampal neurogenesis in the rat. Schizophr Res 2009;113:288-297.

33 Howland JG, Cazakoff BN, Zhang Y: Altered object-in-place recognition memory, prepulse inhibition, and locomotor activity in the offspring of rats exposed to a viral mimetic during pregnancy. Neuroscience 2012;201: 184-198.

34 Hsu D: The dentate gyrus as a filter or gate: a look back and a look ahead. Prog Brain Res 2007;163:601-613.

- 35 Glantz LA, Lewis DA: Decreased dendritic spine density on prefrontal cortical pyramidal neurons in schizophrenia. Arch Gen Psychiatry 2000;57:65-73.

-36 Kolluri N, Sun Z, Sampson AR, Lewis DA: Lamina-specific reductions in dendritic spine density in the prefrontal cortex of subjects with schizophrenia. Am J Psychiatry 2005; 162:1200-1202.

37 Juan LW, Liao CC, Lai WS, Chang CY, Pei JC, Wong WR, Liu CM, Hwu HG, Lee LJ: Phenotypic characterization of C57BL/6J mice carrying the Disc1 gene from the 129S6/SvEv strain. Brain Struct Funct 2013, DOI: 10.1007/ s00429-013-0577-8.
38 Gilmore JH, Jarskog LF, Vadlamudi S: Maternal poly I:C exposure during pregnancy regulates TNF alpha, BDNF, and NGF expression in neonatal brain and the maternal-fetal unit of the rat. J Neuroimmunol 2005;159:106112.

39 Meyer U, Nyffeler M, Engler A, Urwyler A, Schedlowski M, Knuesel I, Yee BK, Feldon J: The time of prenatal immune challenge determines the specificity of inflammation-mediated brain and behavioral pathology. J Neurosci 2006;26:4752-4762.

40 Winter C, Djodari-Irani A, Sohr R, Morgenstern R, Feldon J, Juckel G, Meyer U: Prenatal immune activation leads to multiple changes in basal neurotransmitter levels in the adult brain: implications for brain disorders of neurodevelopmental origin such as schizophrenia. Int J Neuropsychopharmacol 2009;12: 513-524.

41 Alagarsamy S, Phillips M, Pappas T, Johnson KM: Dopamine neurotoxicity in cortical neurons. Drug Alcohol Depend 1997;48:105111.

42 Neumann H, Schweigreiter R, Yamashita T, Rosenkranz K, Wekerle H, Barde YA: Tumor necrosis factor inhibits neurite outgrowth and branching of hippocampal neurons by a rhodependent mechanism. J Neurosci 2002;22: 854-862.

43 Gilmore JH, Fredrik Jarskog L, Vadlamudi S, Lauder JM: Prenatal infection and risk for schizophrenia: IL-1beta, IL-6, and TNFa inhibit cortical neuron dendrite development. Neuropsychopharmacology 2004;29:12211229.
44 Meyer U, Feldon J, Knuesel I, Yee BK, Nyffeler M: Adult brain and behavioral pathological markers of prenatal immune challenge during early/middle and late fetal development in mice. Brain Behav Immun 2008;22:469-486.

45 Tang B, Jia H, Kast RJ, Thomas EA: Epigenetic changes at gene promoters in response to immune activation in utero. Brain Behav Immun 2013;30:168-175

46 Wong RO, Ghosh A: Activity-dependent regulation of dendritic growth and patterning. Nat Rev Neurosci 2002;3:803-812.

47 Whitford KL, Marillat V, Stein E, Goodman CS, Tessier-Lavigne M, Chédotal A, Ghosh A: Regulation of cortical dendrite development by Slit-Robo interactions. Neuron 2002;33: 47-61.

48 Narayan S, Nakajima K, Sawa A: DISC1: a key lead in studying cortical development and associated brain disorders. Neuroscientist 2013; 19:451-464.

49 Kvajo M, McKellar H, Arguello PA, Drew LJ, Moore H, MacDermott AB, Karayiorgou M, Gogos JA: A mutation in mouse Disc1 that models a schizophrenia risk allele leads to specific alterations in neuronal architecture and cognition. Proc Natl Acad Sci USA 2008; 105:7076-7081. 